\title{
LA PROTÉOLYSE DE LA CASÉINE PAR LES ENZYMES INTRACELLULAIRES DE CERTAINES BACTÉRIES
}

\author{
par \\ S. POZNANSKI (1), J. LENOIR et G. MOCQUOT
}

Laboratoire de Recherches de la Chaire de Technologie (I.N.R.A.), Ecole Nationale Supérieure Agronomique de Grignon (S.-et-O.) et Station Centrale de Recherches Laitières et de Technologie des Produits Animanx,

Centre National de Recherches Zootechniques, I.N.R.A., Jouy-en-Josas (Seine-et-Oise)

\section{SOMMAIRE}

L'activité protéolytique des enzymes intracellulaires de quatre souches de bactéries, Streptococcus thermophilus, Lactobacillus bulgaricus et Micrococcus caseolyticus 77 et 79 , a été déterminée sur des substrats à base de caséine à $p H$ 5,6. Les essais ont porté sur les enzymes préparées à partir de chacune des souches microbiennes et sur le mélange correspondant à l'ensemble des souches. Ils ont été conduits avec et sans addition de présure, en présence ou non de 4,5 p. 100 de chlorure de sodium. L'intensité de la pro. téolyse a été estimée par détermination de la teneur en azote non précipitable par le calcium et en azote non protéique, après des temps d'action de $2,6,12$ et 24 jours à la température de $30^{\circ} \mathrm{C}$.

Parmi les endoenzymes essayées, seules celles de $L$. bulgaricus se révèlent douées d'un pouvoir d'hydrolyse notable sur la caséine entière. Les extraits cellulaires de $S$. thermophilus et de $M$. caseolyticus 77 apparaissent plus particulièrement aptes à dégrader les produits intermédiaires de l'hydrolyse de la caséine; ces deux extraits sont, en effet, nettement plus actifs sur le substrat modifié par action de la présure que sur le substrat originel. Les enzymes intracellulaires de $M$. caseolyticus 79 sont peu actives.

Comparée à l'activité protéolytique générale de la présure, celle des endoenzymes bactériennes apparaît relativement faible. Toutefois, on constate une différence de qualité entre les produits d'hydrolyse ; ceux résultant de l'action de la présure sont essen-

(1) Boursier de la F.A.O. en France (Fondation André Mayer). Adresse actuelle : Chaire de Technologie Laitière, Université Agricole, Olsztyn (Pologne). 
tiellement des composés de poids moléculaire élevé, insolubles dans l'acide trichloracétique à 12 p. 100 ; ceux formés par les endoenzymes appartiennent à la fraction non protéique, soluble dans ce réactif. Les endoenzymes bactériennes associées se révèlent plus actives que 'lorsqu'elles agissent isolément. En présence de sel à la dose de 4,5 p. 100 on observe une diminution sensible de l'activité protéolytique des enzymes intracellulaires.

La nature du substrat influe notablement sur l'activité des endoenzymes et sur celle de la présure. La protéolyșe de la caséine native est plus intense que celle de la caséine isoélectrique. La différence est particulièrement nette lorsqu'agissent les endoenzymes de $L$. Bulgaricus, les endoenzymes associées et la présure. Les constituants $\alpha_{\mathrm{s}}$ et $\chi$ sont, eux-mêmes, plus profondément dégradés que la caséine native sous l'action des endoenzymes associées. Par action de la présure, la caséine $\chi$ subit une hydrolyse très prononcée; avec la caséine $\alpha_{S}$, on ne constate aucune libération d'azote non protéique.

\section{INTRODUCTION}

L'hydrolyse de la caséine qui se produit au cours de l'affinage des fromages est un processus complexe en raison, notamment, de l'intervention de nombreux systèmes enzymatiques et de la multiplicité des facteurs susceptibles de modifier l'activité de ceux-ci.

Afin de mieux connaître cette protéolyse, il nous a paru utile d'observer le phénomène dans des conditions contrôlées, en mettant en œuvre les enzymes intracellulaires de certains microorganismes qui peuplent les fromages en voie de maturation.

Amunstad (1950) avait déjà extrait de quelques bactéries lactiques des endoenzymes capables d'hydrolyser la caséine. En étudiant l'action de ces enzymes il a constaté que le $p H$ optimum de leur activité protéolytique est compris entre 6,0 et 6,7 ; il a montré que si les endoenzymes de Streptococcus lactis et de Streptococcus diacetilactis sont relativement peu actives, celles de certaines souches de Betacoccus cremoris sont douées d'un pouvoir protéolytique élevé. Ces résultats venaient à l'appui des travaux de Peterson, Johnson et Price (1948) selon lesquels les protéinases actives du fromage de Cheddar sont en grande partie d'origine microbienne. Toutefois Baribo et Foster (1952), après avoir étudié les protéinases intracellulaires de S. lactis, de L. casei et de Micrococcus freudenreichii soutiennent que les enzymes microbiennes ne comptent que pour une part assez faible dans l'activité des protéinases du fromage.

Van der Zant et Nelson (1953) ont déterminé les caraetéristiques des endoenzymes de S. lactis; ils ont constaté que leur comportement varie avec la nature du substrat. Ils ont notamment 
remarqué que l'activité de la protéolyse est plus intense sur le lait écrémé que sur un substrat à base de caséine ou de lactalbumine. En suivant l'influence du $p H$ sur la protéolyse, ils ont mis en évidence deux optima, un optimum principal à $p H$ et un optimum secondaire à $p \mathrm{H} \quad 5,5$ confirmant ainsi les observations de Baribo et Foster (1952).

Dans notre travail, nous avons suivi, sur un substrat à base de caséine, l'activité protéolytique des endoenzymes préparées à partir de cellules de S. thermophilus, de L. bulgaricus et de Micrococcus caseolyticus. Ces endoenzymes bactériennes ont été essayées isolément et en association, avec et sans addition de présure, en présence ou non de 4,5 p. 100 de chlorure de sodium. La protéolyse a été déterminée à $p \mathrm{H} \mathrm{5,6,pH}$ proche de celui de la plupart des fromages à pâte ferme (Kosikowski et Mocquot, 1958). Quant au choix des espèces bactériennes, il repose sur le fait que $S$. thermophilus et $L$. bulgaricus peuplent les levains lactiques mis en œuvre dans la fabrication des fromages à pâte cuite et que $M$. caseclyticus est présent dans de nombreux types de fromages.

\section{TECHNIQUES EXPERIMENTALES}

\section{Préparation des enzymes endo-bactériennes.}

Les endo-enzymes ont été préparées à partir des souches suivantes: S. thermophilus TJ, L. bulgaricus $\mathrm{LY}_{3}, M$. caseolyticus 77 et 79. Ces souches proviennent de la collection de la Station Centrale de Microbiologie et Recherches Laitières de Jouy-en-Josas,

Les bactéries ont été cultivées sur du lactosérum (obtenu par coagulation du lait par la présure) déprotéiné (après acidification) par chauffage et amené à $p H$ 6,8, puis stérilisé par autoclavage $20 \mathrm{mn}$ à $120^{\circ}$ C. 2,5 litres de lactosérum sont ensemencés avec $125 \mathrm{ml}$ d'une culture de 12 heures sur lactosérum. Le milieu est mis à incuber pendant 24 heures et maintenu au cours de l'incubation à un $p H$ voisin de la neutralité par additions régulières d'une solution stérile de soude. Les températures d'incubation sont les suivantes : $40^{\circ} \mathrm{C}$ pour $S$. thermophilus, $45^{\circ} \mathrm{C}$. pour L. bulgaricus et $30^{\circ} \mathrm{C}$. pour $M$. casseolyticus.

Les cellules bactériennes sont collectées aseptiquement par centrifugation à 7.000 tours $/ \mathrm{mn}$ pendant $45 \mathrm{mn}$. On ajoute au dépôt $25 \mathrm{ml}$ de solution stérile de tampon phosphate $p H 7$ (M/15) et la suspension est conservée une nuit à une température de $0.2^{\circ} \mathrm{C}$.

La libération des enzymes intracellulaires est réalisée par destruction des cellules à l'aide du désintégrateur H. Mickle. Dans chaque cuve de l'appareil on place des billes de verre $(0,11-0,12 \mathrm{~mm}$ de diamètre) et la suspension de cellules en proportions sensiblement égales; l'ensemble occupe $1 / 2$ à $2 / 3$ du volume de la cuve. Le 
mélange est refroidi dans l'eau glacée et soumis à l'agitation pendant un temps de $8 \mathrm{mn}$. Afin d'éviter tout échauffement exagéré les cuves sont refroidies dans l'eau glacée après $2 \mathrm{mn}$ d'agitation. Le taux de destruction des cellules est voisin de 99 p. 100 .

La solution contenant les enzymes endo-bactériennes est ensuite séparée des billes de verre et des débris de cellules par filtration. Après deux lavages sur le filtre avec le tampon phosphate le filtrat est centrifugé $30 \mathrm{mn}$ à 8000 tours, à une température de $0-2^{\circ} \mathrm{C}$. Le surnageant est lyophilisé et le produit sec obtenu conservé à $0^{\circ} \mathrm{C}$ en tubes scellés.

\section{Préparation des caséines.}

La caséine isoélectrique est préparée à partir de lait écrémé par trois précipitations, une première fois à $p H 4,5$, puis deux fois à $p H 4,7$. Après chaque précipitation, la caséine est lavée trois fois à l'eau distillée et dissoute à $p H 7$ par addition lente de soude $\mathrm{N}$.

La caséine "premier cycle ", caséine native, est préparée selon la méthode de Von Hippel et Waugh (1955). La caséine $\chi$ est obtenue en suivant le procédé de McKenzie et Wake (1961), la caséine $\alpha_{\mathrm{s}}$ selon la méthode de Waugh et al. (1960).

Immédiatement après leur préparation les caséines sont lyophilisées et conservées à $0-2^{\circ} \mathrm{C}$.

\section{$3^{\circ}$ Préparation des substrats.}

$20 \mathrm{~g}$ de caséine lyophilisée sont dissous dans une quantité suffisante de tampon acétate de sodium $p \mathrm{H} 5,6(0,25 \mathrm{M})$ pour faire 1 litre de solution. Dans le cas d'un substrat contenant du sel, $45 \mathrm{~g}$ de chlorure de sodium pur pour analyses sont ajoutés après dissolution de la caséine.

Le substrat est réparti en tubes à essai et autoclavé, une première fois $20 \mathrm{mn}$ à $110^{\circ} \mathrm{C}$, puis une seconde fois, après 4 jours, dans les mêmes conditions.

\section{Protocole des essais de protéolyse.}

Chaque série d'essais comporte les combinaisons suivantes : le substrat seul qui constitue le témoin (S), le substrat additionné de l'extrait enzymatique (SE), le substrat additionné de présure (SP) et le substrat additionné à la fois d'extrait enzymatique et de présure (SPE). Avant incubation le substrat est additionné de merthiolate afin d'inhiber les développements bactériens éventuels. On a vérifié que la concentration utilisée, $25 \mu \mathrm{g}$ par $\mathrm{ml}$, n'exerce aucune influence sur l'activité enzymatique des bactéries (Van der Zant et Nelson, 1953), ni sur celle de la présure.

La quantité d'extrait enzymatique ajoutée est de $0,25 \mathrm{mg}$ par ml de substrat. Lorsque l'essai porte sur le mélange des quatre 
extraits des quantités équivalentes de chacun d'eux sont mises en œuvre $(0,062 \mathrm{mg}$ par ml).

La présure utilisée est une présure cristallisée Hansen. Des doses variant de 0,4 à $1 \mu \mathrm{g}$ par $\mathrm{ml}$ de substrat sont ajoutées.

Le merthiolate, les extraits enzymatiques et la présure sont, avant leur addition au substrat, dissous dans l'eau distillée stérile et les mélanges réactionnels sont composés de $23,5 \mathrm{ml}$ de substrat et $0,5 \mathrm{ml}$ de merthiolate auxquels sont ajoutés $1 \mathrm{ml}$ d'eau stérile (essai témoin S), $0,5 \mathrm{ml}$ d'extrait enzymatique et $0,5 \mathrm{ml}$ d'eau (essai SE), $0,5 \mathrm{ml}$ de présure et $0,5 \mathrm{ml}$ d'eau (essai $\mathrm{SP}$ ), $0,5 \mathrm{ml}$ de présure et $0,5 \mathrm{ml}$ d'extrait enzymatique (essai SPE).

Les mélanges réactionnels, placés en tubes à essai, sont maintenus à la température de $30^{\circ} \mathrm{C}$ et la protéolyse est observée après $0,2,6,12$ et 24 jours.

La protéolyse est suivie à l'aide des déterminations suivantes, réalisées en double :

a) Azote correspondant aux protéines précipitables par le calcium (P.P.Ca) après action primaire de la préssure (Garnier, 1963).

A $2 \mathrm{ml}$ de substrat placés dans un tube de centrifugeuse et maintenus à $30^{\circ} \mathrm{C}$ on ajoute $0,1 \mathrm{ml}$ d'une solution de présure contenant $4 \mu \mathrm{g}$ d'enzyme cristallisée. On agite la solution $10 \mathrm{se}-$ condes et après $5 \mathrm{mn}$ on lui ajoute $1 \mathrm{ml}$ d'une solution de $\mathrm{Cl}^{2} \mathrm{Ca}$ $0,09 \mathrm{M}$; on agite, puis, après $15 \mathrm{mn}$, on centrifuge $15 \mathrm{mn}$ à $700 \mathrm{~g}$. Sur le surnageant on dose l'azote par micro-Kjeldahl. L'azote correspondant au P.P.Ca est obtenu par différence entre l'azote total du substrat et l'azote du surnageant.

Lorsque ce traitement est appliqué à un substrat (caséine native ou isoélectrique, etc.) n'ayant pas subi d'action enzymatique préalable, les protéines qui précipitent après l'action successive de la présure (action primaire) et du calcium correspondent à la paracaséine. Mais lorsqu'on a fait préalablement agir sur le substrat soit des enzymes bactériennes, soit la présure elle-même (action protéolytique générale, correspondant à une durée d'action prolongée), les produits qui précipitent par le calcium cessent de correspondre à la paracaséine. Ils sont désignés sous le terme de "Protéines précipitables par le Calcium " (P.P.Ca).

b) Azote non protéique déterminé par précipitation du mélange réactionnel par l'acide trichloracétique à 12 p. 100 (Rowland, 1938) et dosage de l'azote du filtrat par micro-Kjeldahl.

c) Azote ammoniacal déterminé par colorimétrie à $430 \mathrm{~m} \mu$ avec le réactif de Nessler (Hetrick et Whitney, 1949) sur le filtrat trichloracétique à $12 \mathrm{p} .100$.

En outre, après 24 jours d'incubation, sont recherchées la libération d'amines selon la technique de Silverman et Kosikowski (1956) et la production d'hydrogène sulfuré suivant la méthode de Kristoffersen et Nelson (1955). 

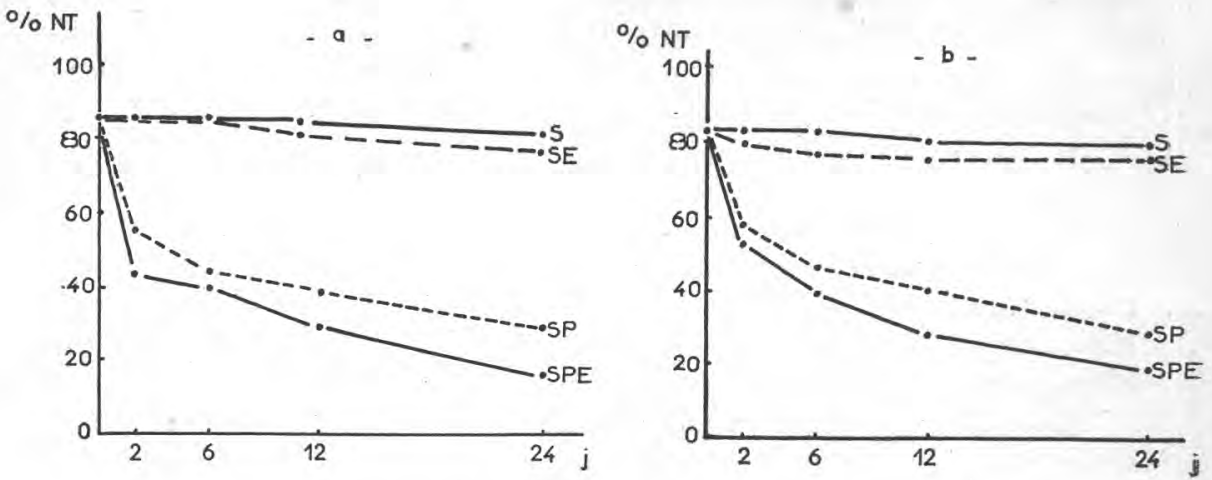

Fig. 1. - Variation de la teneur en azote correspondant aux protéines précipitables par le calcium sous l'action des enzymes intracellulaires de $S$. thermophilus à $p \mathrm{H} 5,6$.

a) Caséine isoélectrique ;

b) Caséine native.

Concentration en caséine : $20 \mathrm{mg} / \mathrm{ml}$.

Concentration en présure (SP et SPE) : $0,5 \mu \mathrm{g} / \mathrm{ml}$.

En abscisses : durée d'action, en jours.

En ordonnées : azote correspondant aux protéines précipitables par le calcium après action primaire de la présure, pourcent de l'azote total.

\section{RÉSULTATS}

\section{I. - Action des endo-enzymes bactériennes sur la caséine entière.}

\section{$1^{\circ}$ Endoenzymes de S. thermophilus.}

En se basant sur le taux des protéines précipitables par le calcium après action primaire de la présure, on constate que les endoenzymes de la souche de $S$. thermophilus ne présentent pas une grande activité protéolytique sur la caséine isoélectrique, ni sur la caséine native (fig. 1). Après 24 jours d'action, l'azote des protéines précipitables par le calcium (P.P.Ca) des deux substrats a seulement diminué de 4 p. 100 (tableau 1). On note cependant que la diminution du taux de P.P.Ca s'amorce dès le deuxième jour avec le substrat caséine native alors qu'elle ne devient appréciable qu'après 12 jours avec le substrat caséine isoélectrique.

L'action protéolytique générale de la présure (Alais et al., 1953) se révèle par contre nettement plus intense et plus rapide sur l'un et l'autre des substrats essayés. La quantité d'azote 


\section{TABLEAU 1}

ACTIVITÉ DES ENDOENZYMes BACTÉRIENNES ET DE LA PRÉSURE SUR LA CASÉINE NATIVE

Azote correspondant aux protéines précipitables par le calcium après action primaire de la présure, \% de l'azote total

\begin{tabular}{|c|c|c|c|c|c|c|}
\hline \multirow{2}{*}{ Enzymes } & \multirow{2}{*}{ Essai (1) } & \multicolumn{5}{|c|}{ Temps d'action en jours } \\
\hline & & 0 & 2 & 6 & 12 & 24 \\
\hline Présure $(0,5 \mu \mathrm{g} / \mathrm{ml}) \ldots$ & $S P-S$ & 0,1 & 25,1 & 36,3 & 40,2 & 52,3 \\
\hline \multirow{2}{*}{ S. thermophilus .... } & $S E-S$ & 0,0 & 3,4 & 5,8 & 4,1 & 4,1 \\
\hline & $S P E-S P$ & 0,0 & 4,9 & 7,5 & 12,1 & 10,0 \\
\hline \multirow{2}{*}{ L. bulgaricus ....... } & $\mathrm{SE}-\mathrm{S}$ & 0,1 & 6,8 & 12,8 & 11,8 & 14,0 \\
\hline & SPE - SP & 0,2 & 4,9 & 0,7 & 12,0 & 0,0 \\
\hline \multirow{2}{*}{ M. caseolyticus $77 \ldots$} & $S E-S$ & 0,0 & 0,7 & 2,8 & 4,1 & 6,5 \\
\hline & $S P E-S P$ & 0,0 & 10,1 & 2,7 & 0,9 & 8,4 \\
\hline \multirow{2}{*}{ M. caseolyticus 79} & $S E-S$ & 0,2 & 1,0 & 1,3 & 0 & 0,9 \\
\hline & $S P E-S P$ & 0 & 2,1 & 2,7 & 0,1 & 0 \\
\hline \multirow{2}{*}{$\begin{array}{l}\text { Association des en- } \\
\text { zymes bactériennes }\end{array}$} & $S E-S$ & 0 & 1,7 & 2,3 & 4,1 & 7,8 \\
\hline & $S P E-S P$ & 0 & 2,1 & 2,7 & 5,5 & 1,9 \\
\hline
\end{tabular}

(1) Les chiffres, sur les lignes horizontales, correspondent à la différence indiquée dans la colonne "Essai ": $\mathrm{SP}-\mathrm{S}$; $\mathrm{SE}-\mathrm{S}$; SPE - SP (pour la signification des lettres $\mathrm{S}, \mathrm{SP}, \mathrm{SE}, \mathrm{SPE}$, se reporter au texte).

P.P.Ca, par rapport à la quantité d'azote total, diminue en effet de 25 à 30 p. 100 après seulement 2 jours d'action, et d'un peu plus de 50 p. 100 après 24 jours.

L'association présure-endoenzymes montre une activité protéolytique sensiblement plus grande que ne le laissent prévoir les 
actions isolées de chacun de ces agents. En comparant les taux de P.P.Ca après 12 jours d'action des endoenzymes on constate que cette activité est près de trois fois plus grande sur les substrats modifiés par action prolongée de la présure que sur les substrats originels.
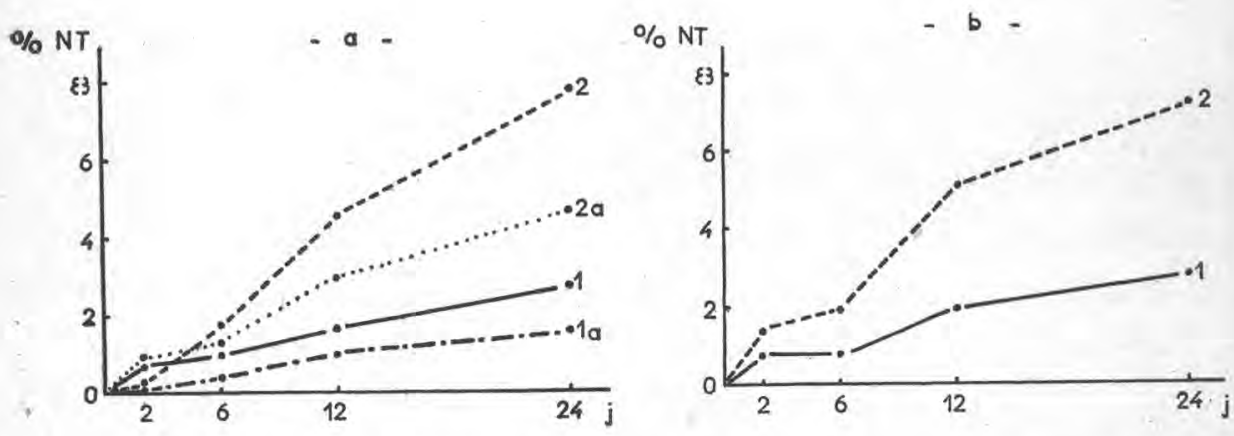

Fig. 2. - Augmentation de l'azote non protéique sous l'action des enzymes intracellulaires de $S$. thermophilus à $p \mathbf{H} \quad 5,6$.

- Courbes 1 : action des enzymes microbiennes seules (SE-S).

- Courbe la : les mêmés en présence de ClNa $(4,5 \%)$.

- Courbes 2 : action combinée des enzymes microbiennes et de la présure (SPE-SP).

- Courbe 2a : les mêmes en présence de ClNa $(4,5 \%)$.

La concentration en caséine et présure, les lettres et signes conventionnels sont les mêmes que dans la figure 1 .

En ordonnées : augmentation de l'azote non protéique en pour cent de l'azote total.

Ces diverses observations sont confirmées par les déterminations de l'azote non protéique (fig. 2). Les enzymes intracellulaires de $S$. thermophilus ne libèrent qu'une faible quantité d'azote non protéique, $2,75 \mathrm{p}$. 100 de l'azote total après 24 jours d'action; sur l'un et l'autre des deux substrats, des cinétiques de protéolyse comparables sont observées. Après l'action de la présure, la libération de l'azote non protéique est plus importante; après 24 jours elle atteint 7,2 p. 100 de l'azote total avec la caséine isoélectrique (tableau 3) et 10,6 p. 100 avec la caséine native (tableau 2). Ainsi déterminée, l'activité protéolytique de la présure apparaît donc singulièrement plus faible que celle qui est mesurée par le dosage de P.P.Ca. Cette constatation rejoint des observations antérieures selon lesquelles la présure libère, essentiellement, aux dépens de la caséine, des composés de poids moléculaire élevé (Sherwood, 1935): En l'absence de présure, l'accroissement de l'azote non protéique sous 1'action des endoenzymes de $S$. thermophilus est 
TABLEAU 2

ACtivité Des endoenzymes bactérienNes et de LA PRÉsure SUR LA CASEINE NATIVE

Azote non protéique $\%$ de l'azote total

\begin{tabular}{|c|c|c|c|c|c|c|}
\hline \multirow{2}{*}{ Enzymes } & \multirow{2}{*}{ Essai (1) } & \multicolumn{5}{|c|}{ Temps d'action en jours } \\
\hline & & 0 & 2 & 6 & 12 & 24 \\
\hline Présure $(0,5 \mu \mathrm{g} / \mathrm{ml}) \ldots$ & $S P-S$ & 0,1 & 3,6 & 5,9 & 8,6 & 10,6 \\
\hline S. thermophilus ... & $\begin{array}{c}S E-S \\
S P E-S P\end{array}$ & $\begin{array}{l}0,1 \\
0,0\end{array}$ & $\begin{array}{l}0,8 \\
1,4\end{array}$ & $\begin{array}{l}0,8 \\
1,9\end{array}$ & $\begin{array}{l}2,0 \\
5,1\end{array}$ & $\begin{array}{l}2,8 \\
7,2\end{array}$ \\
\hline L. bulgaricus . . & $\begin{array}{c}S E-S \\
S P E-S P\end{array}$ & $\begin{array}{l}0,1 \\
0,0\end{array}$ & $\begin{array}{l}3,2 \\
2,7\end{array}$ & $\begin{array}{l}5,9 \\
7,1\end{array}$ & $\begin{array}{l}8,2 \\
8,5\end{array}$ & $\begin{array}{r}10,0 \\
9,6\end{array}$ \\
\hline M. caseolyticus $77 \ldots$ & $\begin{array}{c}\mathbf{S E}-\mathbf{S} \\
\mathbf{S P E}-\mathbf{S P}\end{array}$ & $\begin{array}{l}0,1 \\
0,1\end{array}$ & $\begin{array}{l}0,8 \\
1,9\end{array}$ & $\begin{array}{l}1,2 \\
6,1\end{array}$ & $\begin{array}{r}3,3 \\
10,0\end{array}$ & $\begin{array}{r}4,8 \\
13,4\end{array}$ \\
\hline M. caseolyticus $79 \ldots$ & $\begin{array}{c}S E-S \\
S P E-S P\end{array}$ & $\begin{array}{l}0,0 \\
0,1\end{array}$ & $\begin{array}{l}0,0 \\
0,0\end{array}$ & $\begin{array}{l}0,4 \\
0,4\end{array}$ & $\begin{array}{l}1,2 \\
1,3\end{array}$ & $\begin{array}{l}1,8 \\
1,4\end{array}$ \\
\hline $\begin{array}{l}\text { Association des en- } \\
\text { zymes bactériennes }\end{array}$ & $\begin{array}{r}S E-S \\
S P E-S\end{array}$ & $\begin{array}{l}0,1 \\
0,0\end{array}$ & $\begin{array}{l}1,8 \\
1,9\end{array}$ & $\begin{array}{l}2,8 \\
4,2\end{array}$ & $\begin{array}{l}5,5 \\
7,1\end{array}$ & $\begin{array}{r}7,3 \\
10,4\end{array}$ \\
\hline
\end{tabular}

(1) Cf. note au bas du tableau 1.

sensiblement plus grand que celui observé avec les endoenzymes agissant sur le substrat non modifié; après 24 jours il atteint environ 7 p. 100 de l'azote total dans le premier cas (tableaux 2 et 3, fig. 2) contre 2,75 p. 100 dans le second.

Les essais comportant l'addition de sel au substrat, à la dose de 4,5 p. 100 , montrent que le chlorure de sodium, à cette concentration, diminue très sensiblement les activités protéolytiques des endoenzymes et de la présure. Cependant, là encore, on constate que la libération de l'azote non protéique par les endoenzymes 


\section{TABLEAU 3}

INFLUENCE DU CHLORURE DE SODIUM

SUR L'ACTIVITÉ DES ENDOENZYMES BACTERIENNES ET DE. LA PRÉSURE

Substrat : caséine isoélectrique

Azote non protéique ( $\%$ de l'azote total)

\begin{tabular}{|c|c|c|c|c|c|}
\hline \multirow{2}{*}{ Enzymes } & \multirow{2}{*}{ Essai (1) } & \multicolumn{2}{|c|}{$\begin{array}{l}\text { Sans ClNa } \\
\text { après }\end{array}$} & \multicolumn{2}{|c|}{$\begin{array}{c}\text { Avec } 4,5 \% \text { de } C l N a \\
\text { après }\end{array}$} \\
\hline & & $6 j$ & $24 j$ & $6 j$ & $24 j$ \\
\hline Présure $(0,5 \mu \mathrm{g} / \mathrm{ml}) \ldots$ & $S P-S$ & 4,1 & 7,2 & 2,7 & 5,3 \\
\hline \multirow{2}{*}{ S. thermophilus } & $S E-S$ & 1,0 & 2,7 & 0,4 & 1,5 \\
\hline & SPE - SP & 1,8 & 7,7 & 1,3 & 4,7 \\
\hline \multirow{2}{*}{ L. bulgaricus ...... } & $\mathbf{S E}-\mathbf{S}$ & 1,0 & 2,3 & 0,3 & 0,2 \\
\hline & SPE - SP & 1,4 & 2,2 & 0,6 & 0,9 \\
\hline \multirow{2}{*}{ M. caseolyticus 77} & $\mathbf{S E}-\mathbf{S}$ & 2,2 & 4,3 & 0,7 & 1,5 \\
\hline & SPE - SP & 5,5 & 11,4 & 3,1 & 5,4 \\
\hline \multirow{2}{*}{ M. caseolyticus 79} & $S E-S$ & 0,6 & 1,5 & 0,4 & 0,6 \\
\hline & SPE - SP & 1,2 & 3,9 & 0,5 & 1,5 \\
\hline \multirow{2}{*}{$\begin{array}{l}\text { Association des en- } \\
\text { zymes bactériennes }\end{array}$} & $S E-S$ & 1,8 & 3,7 & 0,5 & 1,2 \\
\hline & SPE - SP & 3,7 & 10,1 & 3,5 & 7,0 \\
\hline
\end{tabular}

(1) Cf. note au bas du tableau 1.

et la présure est plus grande lorsque ces substances sont associées, que lorsque chacune agit seule.

\section{Endoenzymes de L. bulgaricus.}

La nature du substrat mis en œuvre paraît influer très nettement sur l'activité protéolyțique de ces endoenzymes (fig. 3 et 4).

Sur le substrat caséine isoélectrique, elles se révèlent très faiblement protéolytiques. Ainsi, on ne constate aucune modifi- 

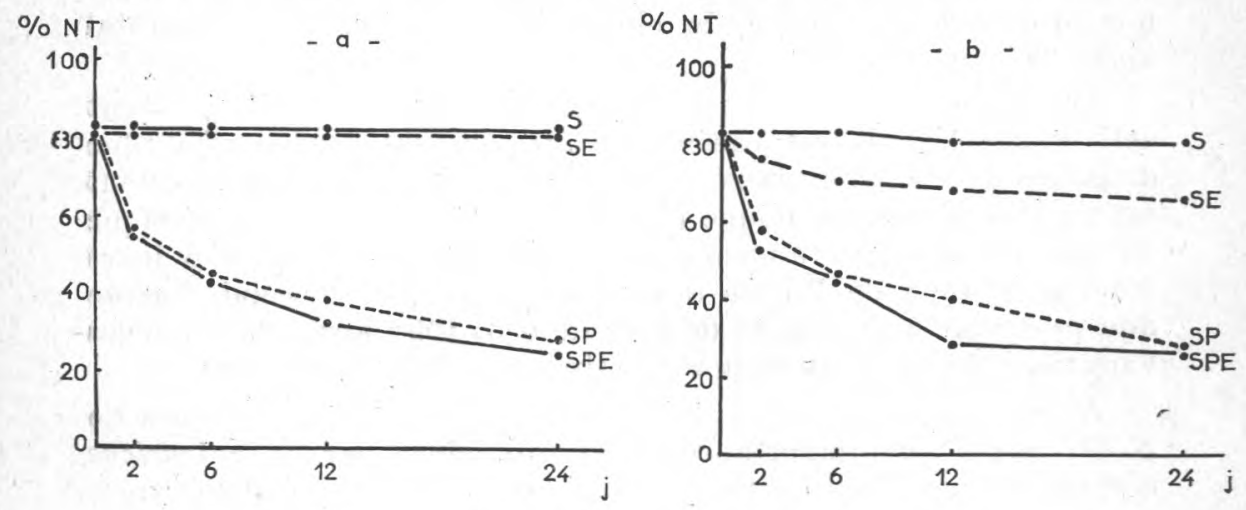

Fig. 3. - Variation de la teneur en azote correspondant aux protéines précipitables par le calcium sous l'action des enzymes intracellulaires de L. Bulgaricus à $p \mathrm{H} 5,6$.

Concentration en présure (essais SP et SPE) : a) $1 \mu \mathrm{g} / \mathrm{ml} ; b, 0,5 \mu \mathrm{g} / \mathrm{ml}$.

Pour les autres indications, se reporter à la légende de la figure 1.
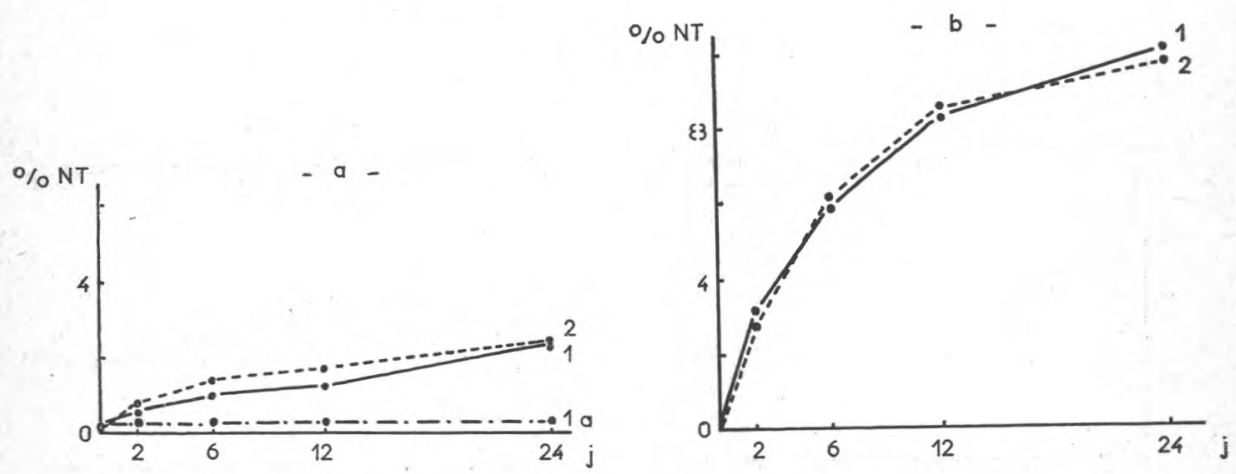

Fig. 4. - Augmentation de l'azote non protéique sous l'action des enzymes intracellulaires de $L$. Bulgaricus à $p \mathrm{H} 5,6$.

Concentration en présure : voir légende de la figure 3.

- Courbes 1 : action des enzymes microbiennes seules.

- Courbe la : les mêmes en présence de ClNa (4,5\%). présure.

- Courbes 2 : action combinée des enzymes microbiennes et de la

Pour les autres indications, se reporter aux légendes des figures 1 et 2. 
cation du taux de P.P.Ca (fig. 3a); toutefois, la libération de l'azote non protéique n'est pas nulle, elle atteint 2 p. 100 de l'azote total après 24 jours (fig. $4 \mathrm{a}$ ).

Les endoenzymes de L. bulgaricus sont par contre relativement actives sur le substrat caséine native. On observe en effet une diminution sensible du taux de P.P.Ca (fig. 3b) et un accroissement net de l'azote non protéique (fig. 4b). Cette activité protéolytique est surtout marquée au cours des premiers jours; après 6 jours d'action le taux de P.P.Ca a diminué de 12 p. 100, celui d'azote non protéique a augmenté de près de $6 \mathrm{p}$. 100 ; après 24 jours les variations de ces taux sont respectivement 14 et 10 p. 100.

A l'inverse de ce qui a été constaté avec les endoenzymes de S. thermophilus, l'importance de la protéolyse par L. bulgaricus n'apparaît pas notablement amplifiée lorsque ses endoenzymes agissent sur le substrat en association avec la présure.

La présence de chlorure de sodium à la dose de 4,5 p. 100 inhibe totalement la libération de l'azote non protéique aux dépens du substrat caséine isoélectrique.

\section{$3^{\circ}$ Endoenzymes de M. caseolyticus : souches 77 et 79 .}

Les enzymes intracellulaires obtenues à partir des deux souches de $M$. caseolyticus présentent, au $p \mathrm{H}$ étudié, des pouvoirs protéolytiques très différents (fig. 5 et 6 ).
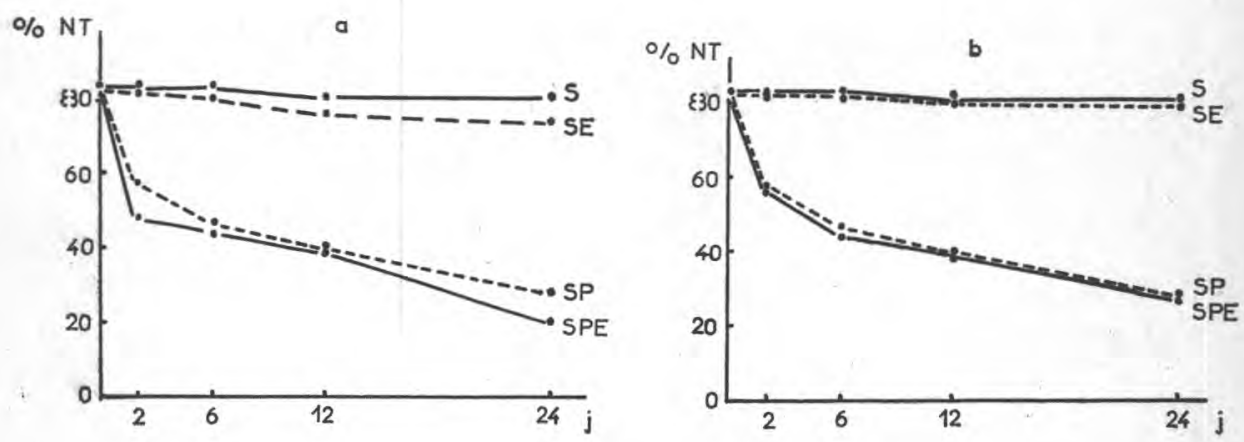

Fig. 5. - Variation de la teneur en azote correspondant aux protéines précipitables par le calcium sous l'action des enzymes intracellulaires de M. Caseolyticus à $p H \quad 5,6$.

a) Souche M. caseolyticus 77 ;

b) Souche $M$. caseolyticus 79 .

Substrat : caséine native, concentration $20 \mathrm{mg} / \mathrm{ml}$.

Concentration en présure (essais SP et SPE) : $0,5 \mu \mathrm{g} / \mathrm{ml}$.

Pour les autres indications, se reporter à la légende de la figure 1 . 

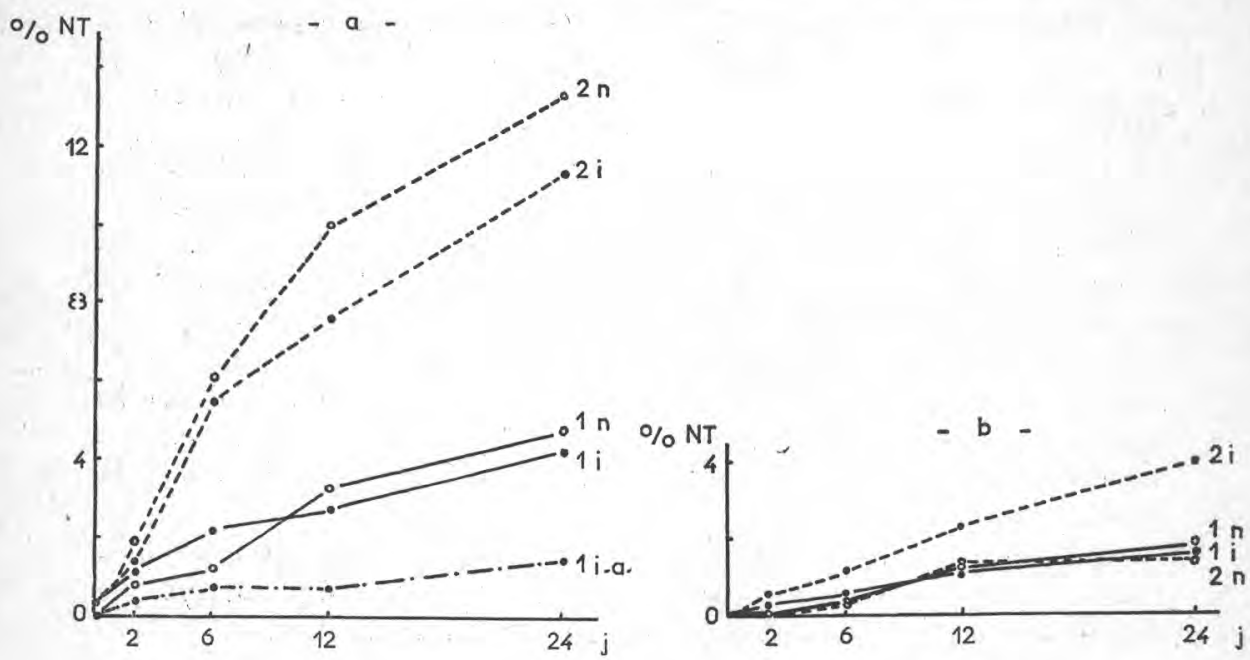

Fig. 6. - Augmentation de l'azote non protéique sous l'action des enzymes intracellulaires de $M$. Caseolyticus à $p H \quad 5,6$.

a) Souche $M$. caseolyticus 77 ;

b) Souche $M$. caseolyticus 79 .

Concentration en caséine : $20 \mathrm{mg} / \mathrm{ml}$.

Concentration en présure (essais SP et SPE) : $0,5 \mu \mathrm{g} / \mathrm{ml}$ pour $M$. caseolyticus 79 sur les deux substrats et $M$. caseolyticus 77 sur la caséine native : $0,4 \mu \mathrm{g} / \mathrm{ml}$ pour $M$. caseolyticus $77 \mathrm{sur}$ la caséine isoélectrique.

- Courbes (i) : substrat caséine isoélectrique.

- Courbes (n) : substrat caséine native :

- 1: action des enzymes microbiennes seules;

- 1a: les mêmes en présence de 4,5 p. 100 de ClNa;

- 2 : action combinée des enzymes microbiennes et de présure.

Pour les autres indications se reporter aux légendes des figures 1 et 2.

Les endoenzymes de la souche 77 (fig. 5a et 6a) sont nettement plus actives sur les deux substrats essayés que celles de la souche 79 (fig. $5 \mathrm{~b}$ et $6 \mathrm{~b}$ ) pour laquelle on n'observe qu'une protéolyse très faible.

La souche 77 libère 4,85 p. 100 d'azote non protéique après 24 jours d'action sur le substrat caséine native; dans les mêmes conditions la souche 79 n'en libère que 1,82 p. 100. En présence de présure, les taux d'azote non protéique libéré sont respectivement 13,36 et 1,38 p. 100 .

L'activité protéolytique de $M$. caseolyticus 77 est comparable à celle de la souche de $S$. thermophilus TJ. En association avec la présure, $M$. caseolyticus 77 protéolyse aussi beaucoup plus 
activement que lorsqu'il agit seul. On remarque cependant que ses enzymes intracellulaires paraissent avoir un pouvoir d'hydrolyse marqué sur les produits de dégradation de la caséine par action de la présure; en effet, lorsqu'elles agissent en association avec la présure, le taux d'azote des P.P.Ca, correction faite de l'action propre de la présure, n'est guère différent de celui observé lors de leur action sur le substrat non modifié. Par contre, dans les mêmes conditions, des différences notables sont constatées sur les taux d'accroissement de l'azote non protéique.

Avec la souche $M$. caseolyticus 77, la protéolyse est comparable sur les deux substrats essayés. Avec la souche $M$. caseolyticus 79 agissant en association avec la présure, le degré de protéolyse est sensiblement plus élevé sur la caséine isoélectrique que sur la caséine native.

On remarque enfin qu'à la concentration de 4,5 p. 100, le chlorure de sodium diminue de plus de 50 p. 100 l'activité protéolytique des endoenzymes des deux souches de microcoques.

\section{$4^{\circ}$ Endoenzymes associées (fig. 7 et 8).}

Les quatre endoenzymes associées présentent une activité protéolytique sensiblement plus grande que la moyenne des activités de chaque enzyme essayée isolément. Ainsi, sur le substrat caséine native et après 24 jours, le taux moyen d'azote non protéique libéré par les enzymes agissant seules est de 4,8 p. 100 ; le taux libéré par les enzymes associées est 7,3 p. 100. En présence de présure ces taux sont respectivement 7,9 et 10,4 p. 100 . Il
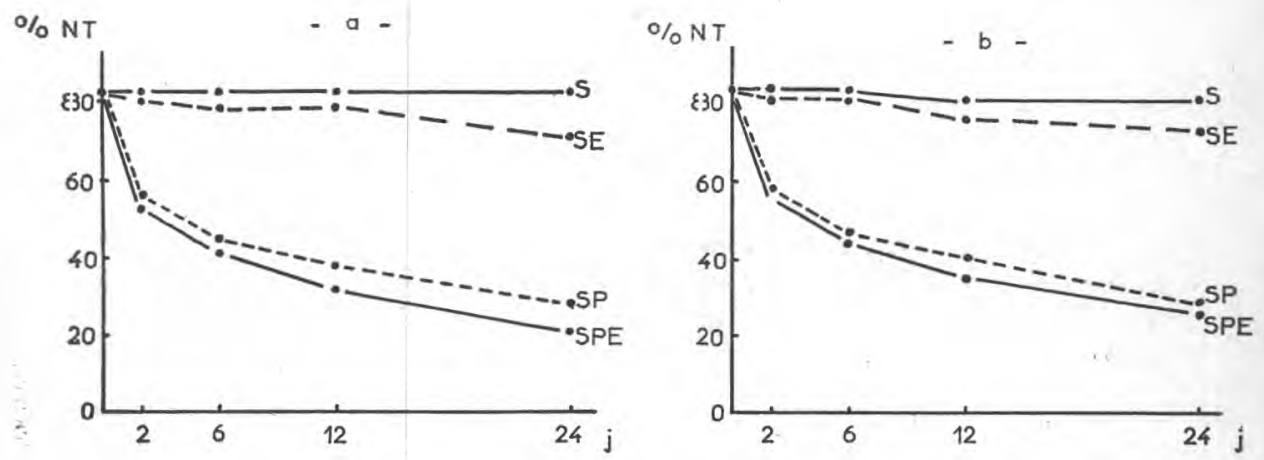

Fig. 7. - Variation de la teneur en azote correspondant aux protéines précipitables par le calcium sous l'action combinée des enzymes intracellulaires de S. Thermophilus, L. Bulgaricus et M. Caseolyticus 77 et 79 à $p \mathrm{H} 5,6$.

Concentration en présure (SP et SPE) : a) $1 \mu \mathrm{g} / \mathrm{ml}$; b) $0,5 \mu \mathrm{g} / \mathrm{ml}$.

Pour les autres indications, se reporter à la légende de la figure 1. 

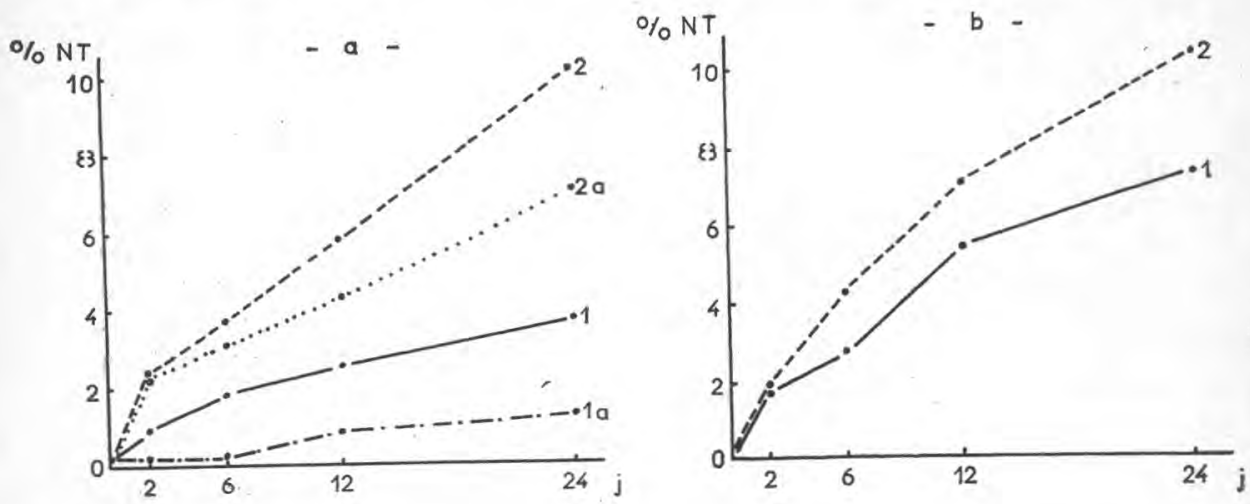

Fig. 8. - Augmentation de l'azote non protéique sous l'action combinée des enzymes intracellulaires de $S$. Thermophilus, L. Bulgaricus et $M$. Oaseolyticus 77 et 79 à $p H \quad 5,6$.

Concentration en présure (essais SP et SPE) : a) $1 \mu \mathrm{g} / \mathrm{ml}$; b) $0,5 \mu \mathrm{g} / \mathrm{ml}$. Pour les autres indications, se reporter à la légende de la figure 2.

apparaît donc que, sur le substrat modifié par action de la présure, l'activité des enzymes associées est nettement favorisée et ce, à un degré du même ordre que pour les enzymes agissant isolément.

L'influence de la nature du substrat caséine est assez sensible. En 24 jours, à partir de la caséine native, les enzymes associées libèrent en effet 7,3 p. 100 d'azote non protéique; à partir de la caséine isoélectrique elles en libèrent 3,7 p. 100. Cette différence dans l'importance de la protéolyse confirme l'observation faite précédemment avec l'endoenzyme de $L$. bulgaricus qui paraît révéler ainsi son activité au sein de l'association.

L'action inhibitrice du sel à la dose de 4,5 p. 100 est également constatée, elle est surtout nette lorsque les enzymes attaquent le substrat en l'absence de présure.

L'activité des endoenzymes associées sur le substrat caséine isoélectrique modifié par action de la présure a fait l'objet des études complémentaires suivantes :

Une première série d'essais comporte l'addition de présure après 12 jours d'action des endoenzymes. Le taux d'azote non protéique libéré par les enzymes intracellulaires après 24 jours, correction faite de l'activité propre à la présure, est de 9,2 p. 100 . Il est encore très nettement supérieur à celui des enzymes agissant sur le substrat en absence de présure (3,7 p. 100) et sensiblement égal au taux d'azote libéré dans l'essai témoin pour lequel le temps d'action de la présure est de 24 jours (10,1 p. 100). La présence de 4,5 p. 100 de chlorure de sodium modifie toutefois les données précédentes. L'azote non protéique libéré par l'action des enzymes 
n'est plus que 3,25 p. 100 dans l'essai comportant l'addition de présure après 12 jours, il atteint $7,1 \mathrm{p} .100$ dans l'essai comportant l'addition de présure dès le début.

Dans une deuxième expérience les enzymes associées sont ajoutées au substrat après 12 jours d'action de la présure. La libération d'azote non protéique par les endoenzymes qui ont agi pendant 12 jours est pratiquement égale à celle qui est observée lorsque les mêmes enzymes agissent pendant 24 jours en présence de présure. Cette constatation confirme l'aptitude des enzymes associées à l'hydrolyse des produits de dégradation formés par action prolongée de la présure sur la caséine entière.

\section{II. - Action des endoenzymes bactériennes sur les caséines $x$ et $\alpha_{\mathrm{s}}$ (fig. 9).}

\section{Caséine $x$.}

La protéolyse de la caséine $x$ par les endoenzymes bactériennes associées est plus importante que celle observée avec la caséine
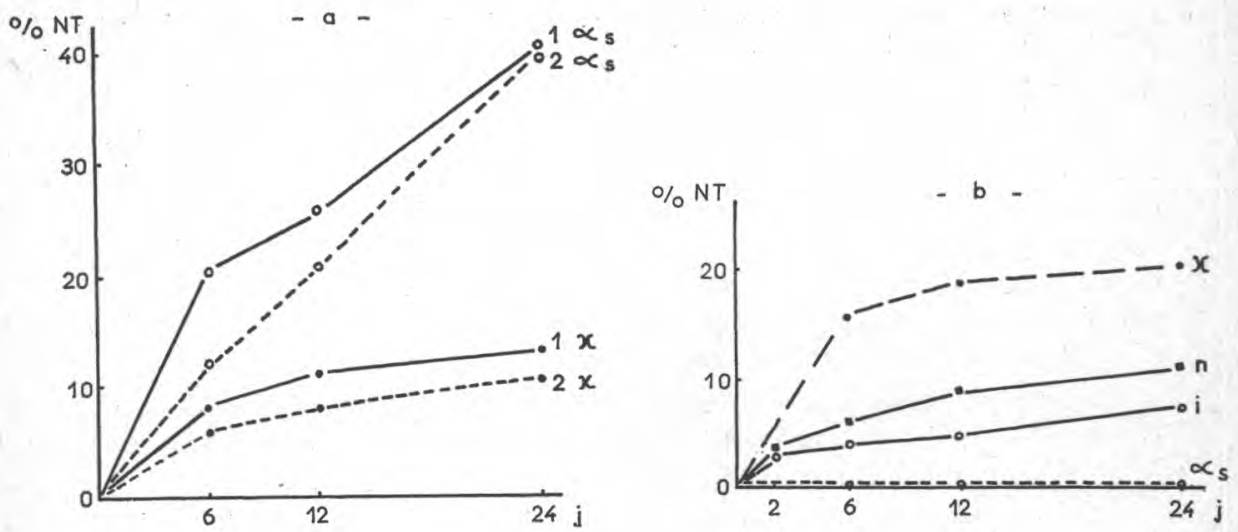

Fig. 9. - Augmentation de l'azote non protéique.

a) Suus l'action de l'association des enzymes intracellulaires;

b) Sous l'action de la présure seule.

Concentration en caséine : $20 \mathrm{mg} / \mathrm{ml}$.

Concentration en présure (essais SP et SPE) : $0,5 \mu \mathrm{g} / \mathrm{ml}$ :

- Courbes $\left(\alpha_{\mathrm{S}}\right)$ : substrat caséine $\alpha_{\mathrm{S}}(p H$ 6,8).

- Courbes $(x)$ : substrat caséine $x(p \mathrm{H} 5,6)$.

- Courbes (n) et (i) : substrats caséine native et caséine isoélectrique $(p \mathrm{H} 5,6)$ :

1 : action des enzymes microbiennes;

2 : action des enzymes microbiennes en présence de présure.

Pour les autres indications se reporter à la légende de la figure 2. 
isoélectrique et avec la caséine native. Après 24 jours d'action, les enzymes libèrent en effet 13,3 p. 100 d'azote non protéique au dépens de ce substrat alors que les taux libérés n'étaient que 3,7 p. 100 pour la caséine isoélectrique et 7,3 p. 100 pour la caséine native.

L'activité protéolytique de la présure se révèle, c'est un fait déjà bien connu, plus intense sur ce substrat que sur les caséines entières. Les taux d'azote non protéique libéré après 24 jours sont respectivement 19,0 p. 100 pour la caséine $x, 7,2$ p. 100 pour la caséine isoélectrique et 10,6 p. 100 pour la caséine native.

Toutefois, contrairement à ce que l'on observe avec les caséines entières modifiées par action de la présure, l'hydrolyse de la caséine $x$ par les endoenzymes n'est pas plus importante lorsque celles-ci agissent en présence de présure. Le taux d'azote non protéique libéré dans ces conditions, correction faite de l'action propre de la présure, est même un peu plus faible, 10,7 au lieu de 13,3 p. 100.

\section{$2 \circ$ Caséine $\alpha_{\mathrm{s}}$.}

La protéolyse de la caséine $\alpha_{s}$ a été déterminée à $p H$ 6,8. Dans ces conditions on constate que les endoenzymes associées libèrent une proportion d'azote non protéique très élevée, cette proportion atteint, en effet, 20 p. 100 de l'azote total après 6 jours et 40 p. 100 après 24 jours d'action.

Sur le substrat additionné de présure on n'observe aucune libération d'azote non protéique même après 24 jours. Tout se passe donc comme si la caséine $\alpha_{\mathrm{s}}$ était insensible à l'action protéolytique générale de la présure, ou comme si les produits de l'hydrolyse éventuelle étaient seulement formés de composés de poids moléculaire élevé, insolubles dans l'acide trichloracétique à 12 p. 100 (Garnier, 1962).

D'après Garnier, Yon et Mocquot (1964) il semble que cette dernière hypothèse soit à retenir puisque, si la présure ne libère pas d'azote soluble dans l'acide trichloracétique à 12 p. 100 , elle semble néanmoins en libérer un peu dans l'acide trichloracétique à 2 p. 100 .

Lorsque les enzymes bactériennes agissent en présence de présure, il semble y avoir un ralentissement de la protéolyse au début; après 6 jours le taux d'azote non protéique libéré n'atteint que 12 p. 100 . Mais au bout de 24 jours la quantité d'azote non protéique formée est identique à celle observée après action des enzymes bactériennes seules soit 40 p. 100 .

\section{III. - Formation des produits ultimes de la dégradation de la caséine: ammoniaque, amines, hydrogène sulfuré.}

Dans les conditions des essais, les endoenzymes bactériennes étudiées ne semblent pas douées d'un pouvoir de désamination. 
On ne constate en effet aucune formation d'ammoniaque, même lorsque la protéolyse du substrat est relativement poussée. On observe au contraire, dans la plupart des essais, une diminution du taux d'azote ammoniacal par rapport à un essai témoin. Le même phénomène est d'ailleurs observé avec les substrats additionnés de présure. Bien que ces variations ne portent que sur des teneurs en $\mathrm{N}$ ammoniacal très faibles $(0, \mathrm{I}$ à 0,2 p. 100 de l'azote total) elles sont cependant appréciables.

Même après 24 jours d'action des endoenzymes, essayées séparément ou en association, on ne constate ni présence d'hydrogène sulfuré, ni présence d'amines en quantités notables. Seules des traces de tyramine ont pu être décelées.

\section{DISCUSSION}

Les enzymes intracellulaires étudiées, à l'exception de celles de $L$. bulgarıcus, se révèlent douées d'un pouvoir protéolytique relativement faible. Les modifications observées dans les taux de protéines précipitables par le calcium sont très légères (tableau 1). La molécule de caséine apparaît ainsi peu sensible à l'action protéolytique des endoenzymes de S. thermophilus et des souches de $M$. caseolyticus. Sur la base d'examens électrophorétiques, Annibaldi (1962) a également constaté qu'une suspension de cellules de $L$. helveticus ne dégrade que très lentement la caséine. Par contre, cet auteur a observé que des suspensions de bactéries connues pour leurs propriétés protéolytiques, comme B. subtrlis et une bactérie du genre Alcaligenes, hydrolysent profondément certaines fractions de la caséine, après 48 à 72 heures d'action seulement.

Il semble donc y avoir une différence non seulement en ce qui concerne l'étendue mais aussi la nature de la protéolyse dont les microorganismes sont capakles. Certains, et notamment ceux caractérisés par un pouvoir protéolytique marqué, sont capables d'attaquer la caséine entière; d'autres, dont l'activité est plus discrète, apparaissent mieux aptes à la dégradation de composés de faible poids moléculaire. Les bactéries qui peuplent les fromages appartiennent, pour la plupart, à ce dernier groupe.

Dans leur étude sur l'action protéolytique des endoenzymes de S. lactis, Van der Zant et Nelson (1953) ont constaté que la libération de la tyrosine était sensiblement plus grande à partir du lait qu'à partir de la caséine ou de la lactalbumine. Plusieurs facteurs peuvent être à l'origine de ce phénomène mais l'on peut penser que les endoenzymes de $S$. lactis dégradent préférentiellement les matières azotées du lait du type protéoses ou polypeptides.

D'ailleurs, divers travaux (Sherwood et Whitehead, 1934 ; Amunstad, 1950 ; Baribo et Foster, 1952) tendent à montrer que 
dans le processus de maturation des fromages les enzymes d'origine microbienne décomposent essentiellement les composés intermédiaires formés par action de la présure sur la caséine. Nos propres observations s'accordent avec cette thèse. On remarque, en effet, que les endoenzymes étudiées libèrent des quantités d'azote non protéique (tableau 2) relativement grandes par comparaison avec les proportions de caséine hydrolysée (tableau 1). On constate également que les enzymes de $S$. thermophilus et de $M$. caseolyticus 77 présentent une activité protéolytique sensiblement plus grande sur le substrat modifié par action prolongée de la présure que sur le substrat originel. Il semble que ces deux systèmes enzymatiques présentent une activité orientée préférentiellement sur les produits intermédiaires de la protéolyse.

On observera cependant que toutes les endoenzymes ne possèdent pas la même aptitude à décomposer les matières azotées provenant de l'hydrolyse de la caséine par la présure. Stadhouders (1962) l'avait d'ailleurs noté sur quelques streptocoques des levains. Nos résultats montrent que les enzymes de $M$. caseolyticus 79 et de $L$. bulgaricus présentent des activités protéolytiques comparables lorsqu'elles agissent sur le substrat, que ce soit en présence ou en l'absence de présure. Il est vrai que les premières ne possèdent qu'un pouvoir d'hydrolyse très faible. Quant aux secondes elles se caractérisent au contraire par une activité marquée sur la caséine native. Cette propriété des endoenzymes de L. bulgaricus a déjà été signalée (Sasaki et Nakae, 1960) mais jusqu'ici on ne semble pas s'y être particulièrement attaché. Cette bactérie pourrait cependant jouer un rôle important dans la maturation des fromages d'Emmental et de Gruyère de Comté.

Un autre point mérite d'être souligné. On sait que dans le processus de maturation des fromages la dégradation des matières azotées est l'œuvre conjointe de la présure et des enzymes d'origine microbienne. Quelles sont les parts respectives de ces agents dans le phénomène de la protéolyse? A la suite des travaux de Sherwood (1935) il est généralement admis que la présure est en grande partie responsable de l'hydrolyse des protéines du fromage. Par son action protéolytique générale elle donnerait naissance à des produits du type protéoses et peptones, mais non aux oligopeptides et acides aminés. La formation de ces derniers serait l'œurre des enzymes bactériennes qui attaquent surtout les protéoses et peptones. Nos résultats ne contredisent nullement ce point de vue. On note en effet que sous l'action de la présure le taux de P.P.Ca diminue de $25 \mathrm{p}$. 100 après 2 jours, de plus de 50 p. 100 après 24 jours; or, parallèlement, les teneurs en azote non protéique n'augmentent que dans des proportions bien plus faibles : 3,6 et 10,6 p. 100. L'activité protéolytique des endoenzymes apparaît beaucoup moins marquée. Sous l'action de $M$. caseolyticus 77, la proportion de caséine hydrolysée en 24 jours est seulement 
6,5 p. 100, mais le taux d'azote non protéique a augmenté de 4,8 p. 100. Toutefois, on ne peut affirmer qu'au cours de la maturation des fromages l'action de la présure puisse être responsable d'une protéolyse aussi marquée que celle observée dans nos essais. Certes, les concentrations de présure que nous avons utilisées sont du même ordre de grandeur que celles habituellement utilisées pour la coagulation du lait de fromagerie, mais il ne reste dans le caillé égoutté qu'une fraction de l'enzyme ajoutée et cette fraction seule intervient en tant qu'agent de maturation.

La présence de chlorure de sodium à la dose de 4,5 p. 100 (concentration voisine de celle trouvée dans la phase aqueuse des fromages), diminue de manière très sensible l'activité protéolytique (tableau 3). Les pourcentages d'azote non protéique libéré après 24 jours en présence de sel sont, par rapport aux témoins sans sel, diminués de 45 à 70 p. 100 selon les souches. L'action de la présure est également affectée par la présence de sel, mais à un degré moindre, le taux d'azote libéré étant seulement diminué d'un peu plus de 25 p. 100 par rapport au témoin. La diminution est également moins marquée lorsque les endoenzymes agissent sur le substrat modifié par action de la présure; les taux d'azote non protéique sont alors diminués de 30 à $60 \mathrm{p}$. 100. Cet effet inhibiteur du sel sur l'activité des enzymes protéolytiques a déjà été constaté par l'un de nous (Poznanski, 1962), avec un ordre de grandeur tout à fait comparable, dans une étude consacrée à la maturation du fromage de Tilsit.

L'association des endoenzymes s'est révélée nettement plus active que ne le laissait prévoir l'action des endoenzymes isolées. Un tel phénomène, assez généralement observé, rend plus difficiles l'interprétation des résultats obtenus à partir de souches pures et leur transposition au niveau des fromages en cours de maturation.

D'ailleurs, cette transposition est d'autant plus incertaine que la nature du substrat influe sur l'activité protéolytique des endoenzymes et de la présure (tableau 4). Il en est ainsi, notam. ment, en ce qui concerne les endoenzymes de $L$. bulgaricus. Celles-ci, nous l'avons vu, sont fortement protéolytiques lorsqu'elles agissent sur la caséine native mais non lorsqu'elles agissent sur la caséine isoélectrique. Les essais réalisés avec les autres enzymes bactériennes, peu actives il est vrai sur la molécule de caséine, ne permettent pas de mettre en évidence des différences appréciables dans le comportement des deux substrats; par contre de telles différences se retrouvent avec les endoenzymes associées et avec la présure.

Endoenzymes associées et présure ont été également essayées sur deux fractions de la caséine entière, la caséine $\alpha_{s}$ et la caséine $x$. Les endoenzymes se révèlent très actives sur la caséine $\alpha_{s}$, beaucoup plus que sur la caséine entière. Le même phénomène est observé, bien qu'à un moindre degré, lorsque les endoenzymes agissent 
TABLEAU 4

INFLUENCE DE LA NATURE DU SUBSTRAT

SUR L'ACTIVIT́́ DES ENDOENZYMES BACTÉRIENNES ET DE LA PRESURE

Azote non protéique ( $\%$ de l'azote total) après 12 jours d'action

\begin{tabular}{|c|c|c|c|c|c|}
\hline \multirow{2}{*}{ Enzymes } & \multirow{2}{*}{ Essai (1) } & \multicolumn{4}{|c|}{ Substrat } \\
\hline & & $\begin{array}{l}\text { Caséine } \\
\text { isoélec- } \\
\text { trique }\end{array}$ & $\begin{array}{l}\text { Caséine } \\
\text { native }\end{array}$ & $\begin{array}{c}\text { Caséine } \\
\alpha_{\mathrm{s}}\end{array}$ & $\begin{array}{c}\text { Caséine } \\
x\end{array}$ \\
\hline Présure $(0,5 \mu \mathrm{g} / \mathrm{ml}) \ldots$ & $S P-S$ & 4,5 & 8,6 & 0,3 & 18,7 \\
\hline S. thermophilus .... & $\begin{array}{c}S E-S \\
S P E-S P\end{array}$ & $\begin{array}{l}1,7 \\
4,5\end{array}$ & $\begin{array}{l}2,0 \\
5,1\end{array}$ & & \\
\hline L. bulgaricus ..... & $\begin{array}{c}S E-S \\
S P E-S P\end{array}$ & $\begin{array}{l}1,2 \\
1,7\end{array}$ & $\begin{array}{l}8,2 \\
8,5\end{array}$ & & \\
\hline M. caseolyticus $77 \ldots$ & $\begin{array}{c}S E-S \\
S P E-S P\end{array}$ & $\begin{array}{l}2,7 \\
7,6\end{array}$ & $\begin{array}{r}3,3 \\
10,0\end{array}$ & & \\
\hline M. caseolyticus 79 & $\begin{array}{c}\mathbf{S E}-\mathbf{S} \\
\mathbf{S P E}-\mathbf{S P}\end{array}$ & $\begin{array}{l}1,1 \\
2,3\end{array}$ & $\begin{array}{l}1,2 \\
1,3\end{array}$ & & \\
\hline $\begin{array}{l}\text { Association des en- } \\
\text { zymes bactériennes }\end{array}$ & $\begin{array}{c}\mathbf{S E}-\mathbf{S} \\
\mathbf{S P E}-\mathbf{S P}\end{array}$ & $\begin{array}{l}2,5 \\
5,8\end{array}$ & $\begin{array}{l}5,5 \\
7,1\end{array}$ & $\begin{array}{l}25,7 \\
20,8\end{array}$ & $\begin{array}{r}11,6 \\
8,2\end{array}$ \\
\hline
\end{tabular}

(1) Cf. note au bas du tableau I.

sur la caséine $x$. Sous l'action de la présure on n'observe aucune libération d'azote non protéique à partir de la caséine $\alpha_{\mathrm{s}}$. Par contre, la caséine $x$ est fortement hydrolysée par la présure et les teneurs en azote non protéique libéré atteignent des valeurs beaucoup plus élevées que celles notées avec tous les autres substrats. Ces observations concernant le comportement particulier des composants de la caséine vis-à-vis de l'activité protéolytique générale de la présure sont à rapprocher du fait que la caséine $x$ constitue, pour cette enzyme, un substrat spécifique, lors de la 
phase primaire de la coagulation du lait. On ne peut toutefois affirmer que la caséine $\alpha_{\mathrm{s}}$ est insensible à l'action protéolytique générale de la présure. Les essais sur ce substrat ont été réalisés à $p \mathrm{H} 6,8$, donc à un $p \mathrm{H}$ assez éloigné du $p \mathrm{H}$ optimum d'activité de l'enzyme qui se situe au voisinage de 4. En outre, si aucune libération d'azote soluble dans l'acide trichloracétique à 12 p. 100 n'est observée, cela peut être dû au fait que les produits d'hydrolyse de la caséine $\alpha_{\mathrm{s}}$ par la présure sont des composés de poids moléculaire élevé, insolubles dans le réactif déprotéinisant.

\section{REMERCIEMENTS}

Nous remercions M. Auclair d'avoir bien voulu mettre à notre disposition les souches de microorganismes utilisées dans cette étude. Nous sommes très reconnaissants à M. Garnier des précieux conseils qu'il nous a donnés concernant la préparation des caséines et nous le remercions de nous avoir procuré la présure cristallisée. Nous adressons enfin nos remerciements à M. Hermier qui a obligeamment mis à notre disposition le désintégrateur Mickle.

\section{SUMMARY}

\section{The proteolysis of casein under the action of certain bacterial endoenzymes.}

The proteolytic activity of the endoenzymes from four bacterial strains, belonging to species Streptococcus thermophilus, Lactobacillus bulgaricus and Micrococcus caseolyticus (strain 77 and 79) on casein substrates, at $p H$ 5.6, has been determined.

The enzymes which were used for the experiments were prepared from each bacterial strain; they were used either separately or in combination, with or without the addition of rennin, with or without the addition of 4,5 p. 100 salt.

The extent of proteolysis has been estimated by determining the nitrogen which does not precipate by calcium and also the non protein nitrogen, after $2,6,12$ and 24 days at a temperature of $30^{\circ} \mathrm{C}$.

Among the various endoenzymes, only those from $L$. bulgaricus give an appreciable proteolytic action on whole casein. The enzymes from $S$.thermophilus and $M$. caseolyticus are more able to decompose the intermediary products formed during casein hydrolysis, as shown by their action on casein to which rennin has been added. The enzymes from $M$. caseolyticus 79 are not very active.

Compared to the "general proteolytic action" of rennin, the activity of the bacterial enzymes appears rather weak. But, when the nature of the products formed during hydrolysis is considered, the situation looks different : while most of the products resulting 
from rennin activity are insoluble in trichloroacetic acid, most of those from the endoenzymes are soluble in the same reagent.

When the endoenzymes are combined together, they seem more active than when they act separately ; their activity decreases markedly in the presence of 4,5 p. 100 salt.

The activity of the bacterial enzymes also varies with the kind of substrate used. Proteolysis is more marked on native casein than on isoelectric casein ; particulary this is the case with $L$. bulgaricus enzymes and with the combined enzymes and rennin.

The $\alpha_{\mathrm{s}}$ and $\chi$ casein are more susceptible to the effect of the combined enzymes than native casein.

\section{RÉFÉRENCES BIBLIOGRAPHIQUES}

C. Alais, G. Mocquot, H. Nitschmann, P. Zahler. 1953. Das Lab und seine wirkung auf das casein der milch. Helv. Chem. Acta, [36], 1955-68.

O. Amunstad, 1950. Recherche sur l'activité protéolytique de la présure et de quelques bactéries lactiques notamment en ce qui concerne la maturation du fromage (en danois). Meddeland fran Statens Mejeri Farsök, Malmö, Lundgren Söners Boktr. [28], 1-170.

S. Annibaldi, 1962. Action caséolytique de quelques microorganismes. $C, R, X V I^{\circ}$ Congrès International de Laiterie, vol. B, 545-553.

L. E. Baribo, E. M. Foster. 1952. The intracellular proteinases of certain organisms and their relationship to the proteinase in cheese. J. Dairy Sci, , [35], 149-160.

J. Garnier. 1962. Etude cinétique de la protéolyse par la présure de la caséine Kappa. Thèse de doctorat. Faculté des Sciences de l'Université de Paris.

J. Garnier. 1963. Etude de l'action de la présure sur la caséine par la méthode titrimétrique à $p H$ constant. Comparaison avec d'autres méthodes. Ann. Biol. Anim. Bioch. Biophys. [3], 71-90.

J. Garnier, S. Yon, G. Mocquot. 1964. Contribution à l'étude de l'association entre la caséine $x$ et la caséine $\alpha_{\mathrm{s}}$ à $p \mathrm{H}$ neutre. Bioch. Biophys. Acta [82], 481-493.

J. H. Hetrick, R. M. WHITNey. 1949. Determination of nitrogen in milk by direct nesslerization of the-digested sample. J. Dairy Sci. [32] 11-112.

F. V. Kosikowski, G. Mocquot. 1958. Progrès de la technologie du fromage, 133. Organisation des Nations Unies pour l'Alimentation et l'Agriculture, Rome.

T. Kristoffersen, F. E. Nelson. 1955. The relationship of serine deamination and hydrogen sulfide production by Lactobacillus casei to Cheddar cheese flavor. J. Dairy Sci. [38], 1319-1325.

H. A. McKenzie, R. G. WAKe. 1961. An improved method for the isolation of $x$ casein. Biochim. Biophys. Acta., [47], 240-242.

M. H. Peterson, M. J. Johnson, W. V. Price. 1948. Proteinase content of Cheddar cheese during making and ripening. J. Dairy Sci., [31], 55-61.

S. PoZNANSKI 1962. The influence of sodium chloride of the transformation of thioaminoacids in Tilsit cheese. C. R.XVI Congrès International de Laiterie, vol, B, 625-633.

R. Sasaki, T. Nakas. 1959. Etudes sur la distribution et les propriétés des bactéries lactiques du lait de vache au Japon. Caractéristiques des 
enzymes protéolytiques produites par quelques bactéries lactiques (en japonais). Jap. J. Zootech. Sci. [30], 7-10 (in Dairy Sci. Abstr., [22], $147,1960)$.

I. R. Sherwood, H. R. Whitehead. 1934. The influence of lactic streptococei on the ripening of Cheddar cheese. J. Dairy Res., [5], 208-222.

I. R. Sherwood. 1935. The role of rennet in the ripening of Cheddar cheesel J. Dairy Res., [6], 204-217.

G. J. Silverman, F. V. Kosokowski. 1956. Amines in cheddar cheese. J. Dairy Sci., [39], 1134-1141.

J. Stadhodders. 1962. The protéolytic activity of rennet and starter bacteria in cheese with reference to bitter flavour. C. R. XVI $I^{\mathrm{e}}$ Congrès International de Laiterie, vol. B, 353-358.

W. C. VAN DER Z Z ANT, F. E. NeLSON. 1953. Characteristics of an endocellular proteolytic enzyme system of Streptococcus lactis. J. Dairy Sci., [36], 1212-1222.

P. H. Von Hippes, D. F. WAUGH. 1955. Casein : monomers and polymers. J. Am. Chem. Soc., [77], 4311-4319.

D. F. Waugh, M. Ludwig, J. M. Gillespie, J. Garnier, E. S. Kleiner, R. W. Nовце. 1960. Certain properties of $\alpha_{\text {s }}$ casein. Fed. Proc., [19], part. I, 337. 